\title{
An Asp to Asn mutation is a toxic trigger in Beta-2 microglobulin: structure and biophysics
}

\author{
Matteo de Rosa ${ }^{1,4}$, Levon Halabelian ${ }^{1}$, Alberto Barbiroli ${ }^{2}$, Martino Bolognesi ${ }^{1}$, \\ Vittorio Bellotti ${ }^{3}$ and Stefano Ricagno ${ }^{1}$ \\ ${ }^{1}$ Dept. of Bioscience, Univ. of Milan (Italy); ${ }^{2}$ Dept. DeFENS, Univ. of Milan (Italy); \\ ${ }^{3}$ Center for Amyloidosis and Acute Phase proteins, UCL, London (UK); ${ }^{4}$ CNR \\ Biophysics Institute, Milano, Italy. \\ Address for correspondence: Stefano Ricagno, Department of Biosciences, University \\ of Milan, 20133 Milano, Italy. E-mail: stefano.ricagno@unimi.it
}

Background. Beta-2 microglobulin $(\beta 2 \mathrm{~m})$ is part of the Major Histocompatibility Complex Class I (MHC I) and when monomeric becomes an aggregation prone protein that is responsible for a human disorder known as dialysis related amyloidosis. In 2012 Valleix et al. described a new familial systemic amyloidosis: an unreported $\beta 2 \mathrm{~m}$ mutant $(\mathrm{D} 76 \mathrm{~N})$ is the etiological agent of such disease. Main symptoms were chronic diarrhoea, loss of weight and polyneuropathy: large amyloid deposits were found in internal organs. From the biophysical point of view, the $\mathrm{D} 76 \mathrm{~N} \beta 2 \mathrm{~m}$ is much less stable and more amyloidogenic than wt $\beta 2 \mathrm{~m}$; however, its crystal structure reveals very minor conformational changes compared with the wt protein [1].

Material and methods. $\beta 2 \mathrm{~m}$ gene was mutagenized by side directed mutagenesis. Proteins were refolded, purified and crystallised according to previously reported protocols [2]. Protein stability was evaluated by Far-UV circular dichroism (CD) as in [3]; protein aggregation propensity was assessed by thioflavin $\mathrm{T}$ fluorescence at 450 nm.

Results. The Unfolded Protein Response (UPR) in the ER should target an unstable protein such the $\mathrm{D} 76 \mathrm{~N}$ variant for degradation, however this is not the case. In order to understand what is the role of the $\mathrm{MHC} \mathrm{I}$ in the protection of $\mathrm{D} 76 \mathrm{~N}$ variant against UPR degradation, the MHC I bearing the D76N mutation have been investigated and evaluated structurally and biophysically. All data indicate that the interactions between $\beta 2 \mathrm{~m}$ and the heavy chain of the MHC I yields to a highly stable complex regardless the stability of the $\beta 2 \mathrm{~m}$ variant in the monomeric state [4]. Therefore the D76N variant is effectively protected from UPR degradation when it is part of the MHC I.

To understand the chemical bases of $\mathrm{D} 76 \mathrm{~N}$ instability and aggregation propensity, two sets of single $\beta 2 \mathrm{~m}$ mutants have been prepared: (I) all Asp were mutated to Asn; (II) Asp76 was mutated to several residues with different chemical properties.

The six Asp in $\beta 2 \mathrm{~m}$ sequence have been mutated to Asn (D-to-N mutants): their thermal stability and aggregation propensity closely resemble those of wt $\beta 2 \mathrm{~m}$; from the structural point of view only the $\mathrm{D} 38 \mathrm{~N}$ mutation triggers non-negligible conformational changes, however the structural rearrangements observed in the C-D region do not correlate with an increased aggregation propensity [5]. In summary, these data indicate that the loss of a negative charge is not sufficient to explain the amyloidogenic properties of the D76N mutant. 


\begin{tabular}{|l|c|c|c|}
\hline & Tm $\left({ }^{\circ} \mathrm{C}\right)$ & Aggregation & $\begin{array}{c}\text { R.M.S.D. } \\
(\mathrm{A})\end{array}$ \\
\hline wt $\beta 2 \mathrm{~m}$ & 63.1 & - & - \\
\hline D34N & 61.8 & - & n.d. $^{*}$ \\
\hline D38N & 60.2 & - & 3.44 \\
\hline D53N & 63.6 & - & 0.17 \\
\hline D59N & 60.7 & - & 0.10 \\
\hline D76N & 53.2 & + & 0.60 \\
\hline D96N & 64.7 & - & n.d. $^{*}$ \\
\hline D98N & 65.2 & - & 0.15 \\
\hline
\end{tabular}

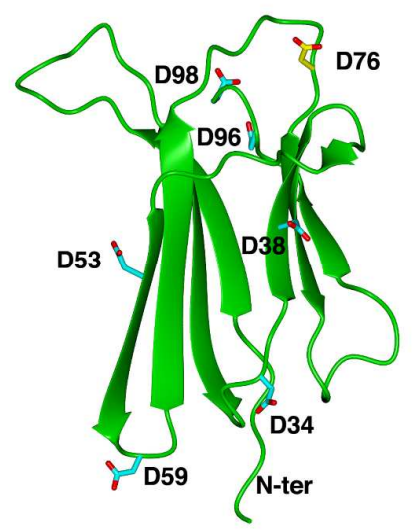

Figure 1. (Left) Table listing the $\beta 2 \mathrm{~m}$ D-to-N mutants and for each of them the following information is reported: the melting temperature monitored by $\mathrm{CD}$; the aggregation propensity; the root mean squared deviation, over $97 \mathrm{Ca}$ atoms, between the wt $\beta 2 \mathrm{~m}$ structure and the ones of the mutants. (* not determined) (Right) Crystal structure of wt $\beta 2 \mathrm{~m}$ highlighting the position of the seven Asp residues.

The second set of mutants focuses on the position 76: four mutants have been prepared: D76A, D76H, D76E, D76K. Although data are preliminary and their analysis is still in progress, it seems that any substitution in the 76 position affects to great extent both stability and amyloidogenicity of $\beta 2 \mathrm{~m}$ in solution without major impact on its structure.

Discussion. Our data clearly indicate that position 76 is very crucial to determine $\beta 2 \mathrm{~m}$ thermal stability and aggregation propensity and only an extensive interaction such the one within the MHC I is able to compensate the loss in stability due to the D76N mutation.

Declaration of interest. The authors report no conflict of interest.

References. 1.Valleix S, Gillmore JD, Bridoux F, Mangione PP, Dogan A, Nedelec B, et al. Hereditary systemic amyloidosis due to Asp76Asn variant beta2microglobulin. N Engl J Med. 2012;366(24):2276-83.

2. Esposito G, Ricagno S, Corazza A, Rennella E, Gumral D, Mimmi MC, et al. The controlling roles of Trp60 and Trp95 in beta2-microglobulin function, folding and amyloid aggregation properties. Journal of molecular biology. 2008;378(4):88595 .

3. Santambrogio C, Ricagno S, Colombo M, Barbiroli A, Bonomi F, Bellotti V, et al. DE-loop mutations affect beta2 microglobulin stability, oligomerization, and the low-pH unfolded form. Protein Sci. 2010;19(7):1386-94.

4. Halabelian L, Ricagno S, Giorgetti S, Santambrogio C, Barbiroli A, Pellegrino $\mathrm{S}$, et al. Class I Major Histocompatibility Complex, the Trojan Horse for Secretion of Amyloidogenic beta2-Microglobulin. The Journal of biological chemistry. 2014;289(6):3318-27.

5. de Rosa M, Barbiroli A, Giorgetti S, Mangione PP, Bolognesi M, Ricagno S. Decoding the Structural Bases of D76N ss2-Microglobulin High Amyloidogenicity through Crystallography and Asn-Scan Mutagenesis. Plos One. 2015;10(12):e0144061. 